\title{
A Distributed Data Mining System for a Novel Ubiquitous Healthcare Framework
}

\author{
M. Viswanathan, T.K. Whangbo, K.J. Lee, and Y.K. Yang \\ College of Software, Kyungwon University, Seongnam-Si, \\ Kyunggi-Do, South Korea \\ \{murli, , tkwhangbo, 1kj9731, ykyang\} @kyungwon.ac.kr
}

\begin{abstract}
Recent years have shown impressive growth in the development of ubiquitous healthcare (u-Healthcare) systems which aim for the next generation in e-Health services and associated research. Such systems are primarily being designed to provide emergency and preventive healthcare to citizens anywhere/anytime using wired and wireless mobile technologies. Data mining is an indispensable aspect of such systems and represents the process of analyzing databases to extract hidden knowledge and relationships. This paper introduces and studies the development framework of a prototype ubiquitous healthcare system initiated by the South Korean government. Preliminary results with a distributed data mining system are presented in the context of a larger future integration with the ubiquitous healthcare framework.
\end{abstract}

Keywords: Distributed Data Mining, Ubiquitous Healthcare, Decision Supporting System, Healthcare Data, Data Classification.

\section{Introduction}

The increase in aging population in several countries has highlighted the need for efficient and preventive healthcare for the elderly. Ubiquitous healthcare is the next step in the integration of information technology with healthcare services and refers to the access to healthcare services at any time and any place for individual consumers through mobile computing technology. These u-Healthcare systems provide enhanced services including real-time patient data collection and monitoring for emergency care as well as preventive health recommendations. The vast amount of data collected from the distributed users of $\mathrm{u}$-Health services results in a growing need for analyzing them across geographical lines using distributed and parallel systems. Implementations of data mining techniques on high-performance distributed computing platforms are moving away from centralized computing models for both technical and organizational reasons [11].

In this paper, we present and discuss the designed prototype for a ubiquitous healthcare system that will provide advanced patient monitoring and health services. Subsequently we introduce and present empirical analysis of a preliminary distributed data mining system. The integration of such a distributed mining system is studied in the context of the decision support framework for our ubiquitous healthcare system. 


\section{2 u-Healthcare Initiatives and Challenges}

There are several ubiquitous challenges in the development of such healthcare frameworks and systems. These include:

- $\quad$ issues of security and privacy related to information transfer through unsecured infrastructure, potentially lost or stolen devices, legal enforcement and other scenarios;

- determining current context and user activity in real-time and locating context dependent information such as automatic discovery of services based on user health needs;

- development of low-power sensors to monitor user context and health condition;

- information management through development of techniques to collect, filter, analyze and store the potentially vast quantities of data from widespread patient monitoring and applying privacy preserving data mining at several levels;

- $\quad$ simple patient interaction systems to provide guidance, feedback and access to medical advice in acute situations;

- Adaptable network infrastructures to support large-scale monitoring, as well as real-time response from medical personnel or intelligent agents.;

- integration of specialized local u-Health architectures for unified data access and connection to National grids;

A growing number of ubiquitous healthcare projects are being pursued by large enterprises owning healthcare related companies and government bodies. MobiHealth project [2] is a mobile healthcare project supported by the EC with countries such as Netherlands, Germany, Spain and Sweden participating in it, and companies such as Philips and HP are providing technical support. EliteCare[3], is an elderly care system developed in the USA that monitors patients using various sensors and provides emergency and health information services. Tele-monitoring service[4] is being developed by the Philips Medical system, where centers analyze data that is collected from homes and transmitted by biomedical signal collection devices, and provide health management and related information. CodeBlue[5] is a sensor network based healthcare system being developed to treat and deal with emergencies, rehabilitation of stroke patients, and in general, to use health signal data in addition to hospital records in real time treatment decisions. The UbiMon[6] project which stands for Ubiquitous Monitoring Environment for Wearable and Implantable Sensors is studying mobile monitoring using sensors and real-time biomedical data collection for long time trend analyses. The Smart Medical Home[7] aims to develop a fully integrated personal health system with ubiquitous technology based on infrared and bio sensors, computers, video cameras and other devices. Sensor data is collected and transmitted to a center for further analysis and preventive care.

\section{U-Healthcare System Framework}

The components of the ubiquitous system prototype are summarized in this section. A system user in this paper refers to a patient who has a contract with a provider to use the ubiquitous healthcare services and regularly receives medical treatment at a hospital. 
Fig. 1 shows an overview of the ubiquitous healthcare service framework as suggested in this paper.

The user wears a sensory device, provided by the hospital, on his wrist. The sensor regularly transmits collected data to a healthcare center through networking or mobile devices, and the transmitted data is stored at the u-healthcare center. In the center, monitoring staff are stationed to answer the user' queries, monitor his biomedical signals, and call an emergency service or visit the patient to check his status when an abnormal pattern is detected. The hospital monitors the collected data and judges the patient's status using the collected biomedical signals in his periodic checkup.
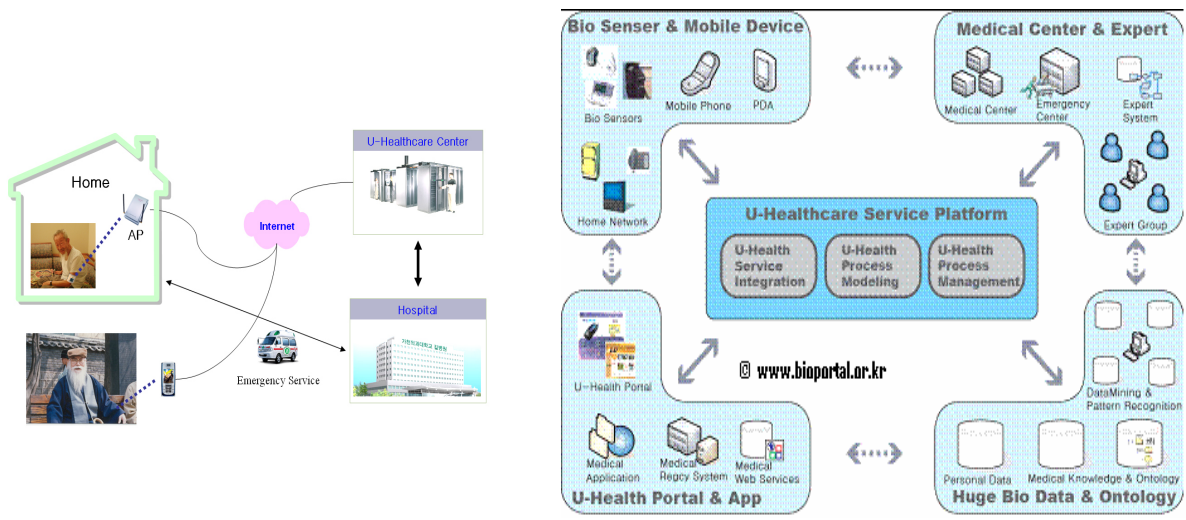

Fig. 1. Ubiquitous Healthcare Framework

\subsection{Biomedical Signal Collection and Transmission}

A sensor attached to a user's wrist collects data such as the user's blood pressure, pulse, and orientation and transmits the collected data to the user's mobile phone or access point (AP) at home using a wireless ZigBee device. Zigbee complements Bluetooth's weaknesses, provides multi hopping, and has low power consumption [8]. Biomedical signals can be collected while moving in and out of the user's residence. The data collected inside of the house is sent to the AP in the house using Zigbee module. The AP stores the collected data and sends it regularly to the data storage at the healthcare center. When the user is outside of the house, the sensor sends the collected data to the user's mobile phone and then using CDMA module of the mobile phone, transmits the data to the center.

\subsection{Healthcare Center}

The healthcare center has two primary roles. First, it provides storage and management for the biomedical data collected from the users, and second, it monitors the users' health status and takes appropriate emergency or preventive action when required. A database server in the healthcare center stores and manages data including the medical, personal, family and other information for all registered users as well as biomedical signals collected from them. This data is used for real-time monitoring of users in case 
of emergencies and is also useful in periodic checkups. The healthcare center also includes personnel who are stationed to keep monitoring users' health status and provide health information as well.

\subsection{CDSS (Clinical Decision Support System)}

The CDSS supports long-term and short-term decision making processes by using models from distributed data mining, developing alternative plans and performing comparison analysis. In the short-term it assists in optimal planning to solve various decision making problems confronted in emergencies by utilizing the biomedical signals. The goal of this system is to provide an information system environment where a decision maker can solve problems easily, accurately and promptly such that users are benefited. The CDSS needs to be integrated with a distributed data mining system that can provide global models.

\subsection{Emergency Response}

Emergencies in a U-health framework require robust and quick recognition followed by an efficient emergency response. In this framework we employ a three pronged emergency recognition drive. Firstly, personnel monitoring the streaming biomedical data may detect abnormal signs and check user through phones or visits. Secondly, abnormal signs are also detected while mining the biomedical data collected over a period by the CDSS. Lastly, motion detectors mounted on sensors detect occurrence of falls and erratic movement.

The emergency management system uses a variety of hardware and software components that aim to improve emergency counteractions at the appropriate time and lower preventable deaths. This includes portable personal terminals comprising of RFID tags, portable RFID readers, an ambulance information system, a hospital information system and a healthcare information system. The efficiency of the treatment in emergency rooms is increased by using RFID tags and readers. Since the system is well integrated it also transfers patient information in real-time to hospitals, and therefore medical teams who will provide treatment during emergencies can be well-prepared.

\subsection{Remote Monitoring System}

With increasing urbanization, shrinking of living space and shifting concepts of the family, elderly people often tend to live alone without any assistance at home. In such cases prompt responses are most important when a medical emergency occurs. The remote monitoring system uses cameras for checking current situation when an abnormal sign is detected by sensory devices. There may be signals that cannot be detected even with motion detectors mounted on sensors, or false alarms may occur. In these cases, the situations can be checked using in-house video cameras. The remote monitoring system is not only a management system for patient monitoring but aims for general health improvement of consumers through prevention of diseases, early detection, and prognosis management [10]. 


\section{Healthcare Decision Support with Data Mining}

Table 1 presents the several levels of data mining applicable in such a healthcare framework. In this paper we consider the scenario [12] where a hospital needs to analyze a very large database with real-time patient sensor data. Due to processing and memory limits the database is partitioned and sent to individual machines to be processed. In this section we discuss how distributed data mining plays an important role within the CDSS component of the ubiquitous healthcare system.

Table 1. Levels of data mining

\begin{tabular}{ll}
\hline Low level & $\begin{array}{l}\text { Sensor data mining for emergency detection } \\
\text { Sensor data fusion for decision support }\end{array}$ \\
\hline \multirow{3}{*}{ Hospital-Based } & $\begin{array}{l}\text { Periodic trend analysis \& wellness recommendations } \\
\text { Analysis of patient groups for drug effects, CRM and indirect factors } \\
\text { Recovery analysis, drug interaction and other studies like allergy cause } \\
\text { and detection }\end{array}$ \\
\hline Global level & $\begin{array}{l}\text { Clustering and classification analysis for epidemics and other implicit } \\
\text { patterns }\end{array}$ \\
\hline
\end{tabular}

\subsection{CDSS and DDM}

In a ubiquitous healthcare framework DDM systems are required due to the large number of streams of data that have a very high data rate and are typically distributed. These need to be analyzed/mined in real-time to extract relevant information. Often such data come from wirelessly connected sources which have neither the computational resources to analyze them completely, nor enough bandwidth to transfer all the data to a central site for analysis. There is also another scenario where the data collected and stored at a center needs to be analyzed as a whole for creating the dynamic profiles. The preliminary empirical analysis with the prototype distributed data mining system discussed in this paper is suited towards this latter situation. The integration of the CDSS component of the ubiquitous healthcare framework with such a DDM is important.

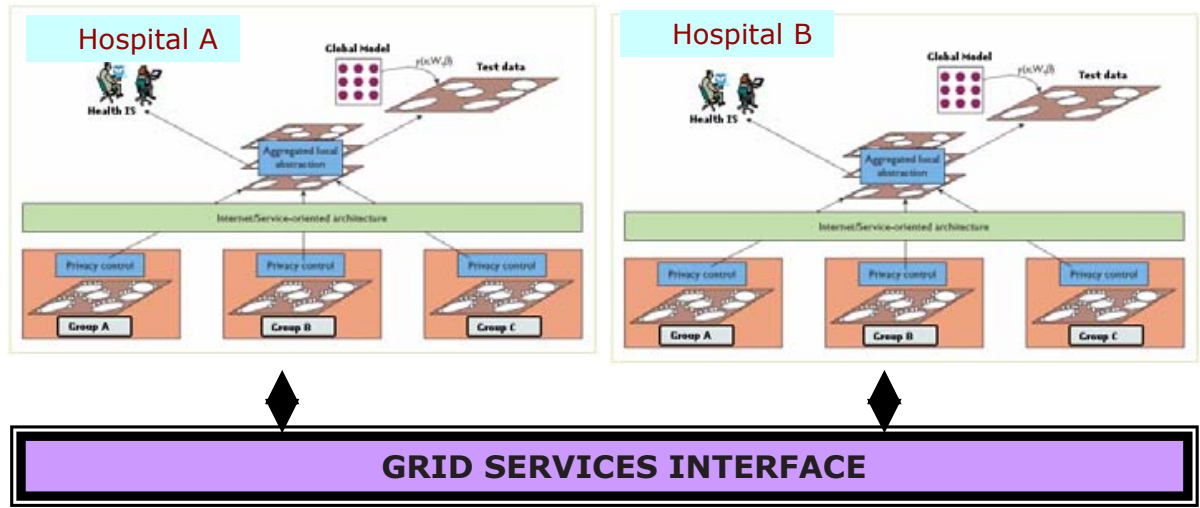

Fig. 2. Local to Global Mining 
Data mining techniques used in the decision making system divide patients into groups. As a collection of patients have their own characteristics, they should be divided properly, and group properties are found through applying cluster analysis modeling techniques and searching created groups in the group analysis step. Secondly, causal models are developed for health patterns using mining techniques. Finally, a dynamic profile of the patient can be created using past history and domain knowledge in conjunction with sensory data. Each patient's risk rate is calculated by a system reflecting mining results, and administrators can see patients' risk rankings from the risk rates and give priority to patients with higher rates.

\subsection{Distributed Data Mining Architecture}

This section outlines a prototype system for DDM applicable in the scenario described above. For a detailed exposition of this system and results see [16]. The DDM system is built from various components as seen in figure 3. The DDM system takes collected biomedical hospital data and using SNOB [13], a mixture modeling tool, partitions it to clusters. The clusters get distributed over the LAN using MPI [14]. Data models are developed for each cluster dataset using the classification algorithm C4.5 [15].

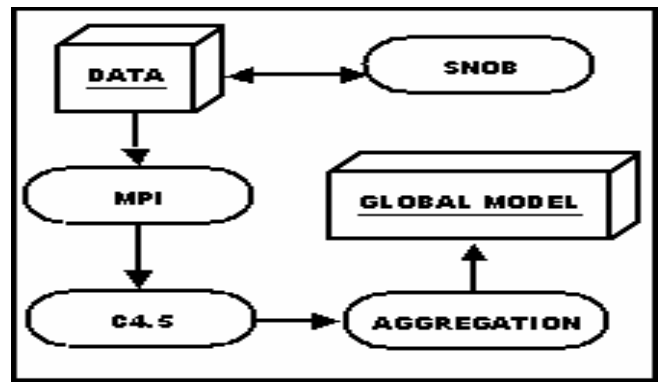

Fig. 3. DDM System Components

Finally the system uses a voting scheme to aggregate all the data models. The final global classification data model comprises of the top three rules for each class (where available). Note that MPI is used in conjunction with the known maximum number of hosts (line 4) to classify the clusters in parallel using the $\mathrm{C} 4.5$ classification algorithm. If the number of clusters exceeds the available number of hosts then some hosts will classify multiple clusters (using MPI). Also the aggregation model scans all Rule files (line 7) from all clusters and picks the best rules out of the union of all cluster rule sets. During the classification phase we have also classified the original dataset and produced rules modeling this data. To finally ascertain if our DDM system is efficient we compare our global model to this data model from the un-partitioned database. We compare the top three rules for each class from this model with our rules from the global model. If a global model is over $90 \%$ accurate in comparison to the data model from the original database we consider this as a useful result. 


\subsection{Preliminary Results}

The DDM system was tested on a number of real world datasets in order to test the effectiveness of data mining and the predictive accuracy. Further empirical analysis can be studied from [16]. In this section we present the DDM system performance results on the 'Pima-Indians-Diabetes' dataset from the UCI KDD Archive [17]. In order to study the usefulness of the system we compare the top three rules for each class from the partition-derived classification rules and rules from the original dataset. The aim of this testing is to find out the effect of our clustering process in partitioning, to the efficiency of our classification model and its predictive accuracy. We apply an error threshold, average error rates of rules from partitions greater then $10 \%$ of that of the corresponding original rules is an undesirable result.

We can observe in figure 4 and other comparisons that the rules from partitions and original rules satisfy our error threshold. In general the distributed data mining system offers useful performance in the presence of a number of factors influencing the predictive accuracy. However many improvements and further research is needed in order to optimize the DDM system.

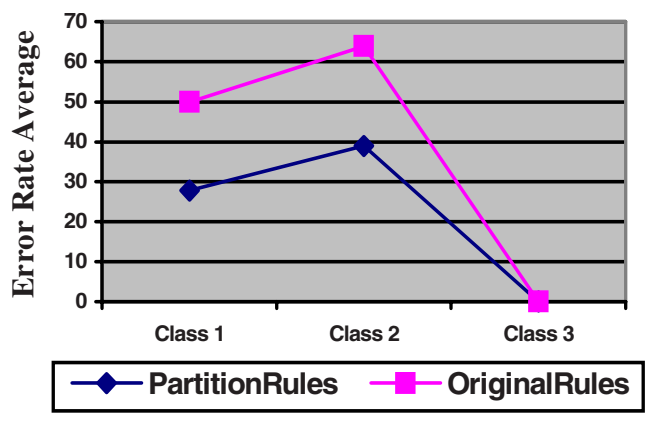

Fig. 4. Predictive Accuracy Comparison

\section{Conclusions and Future Challenges}

As the elderly population constitutes a larger proportion of the aging society, providing quality long term care becomes an increasingly critical issue over the world. Our research aims to enable a patient-centric ubiquitous healthcare environment instead of the existing hospital-centric approach. The use of traditional verification-based approaches to analysis is difficult when the data is massive, highly dimensional, distributed, and uncertain. Innovative discovery-based approaches to health care data analysis with the integration of distributed data mining techniques warrant further attention.

This paper commences by describing a ubiquitous healthcare framework designed to provide consumers with freedom from temporal and spatial restrictions in their access to professional and personalized healthcare services anytime and anywhere - even outside of the hospital. Components of the system framework are discussed in brief. A prototype distributed data mining system is introduced with results from preliminary 
experiments on data. The plausibility of integrating such a DDM system with the clinical decision support component (CDSS) of the ubiquitous healthcare frameworks is highlighted.

\section{References}

1. United Nations Population Division Publications. UN World Population Ageing 1950 2050: http://www.un.org/esa/population/publications-/worldageing19502050 (2002)

2. MobileHealth: http://www.mobihealth.org/

3. EliteCare: http://www.elitecare.com/index.html

4. Tele-Monitoring: http://www.medical.philips.com

5. CodeBlue: http://www.eecs.harvard.edu/ mdw/proj/codeblue

6. Smart Medical Home. http://www.futurehealth.rochester.edu/smart_home/

7. J. Hill, M. Horton, R. Kling, L. Krishnamurthy: The Platforms enabling Wireless Sensor Netowrks. Communications of the ACM, Vol. 47 (2004) 41-46

8. Jardine, I. and Clough K.: The Impact of Telemedicine and Telecare on Healthcare. Journal of Telemedicine and Telecare, Vol. 5, Supplement 1 (1999) 127-128

9. Smithers C. R. and Hill N.: Options for Wireless Technology in Telemedicine and Telecare Applications. Journal of Telemedicine and Telecare, Vol. 5, Supplement 1 (1999) 138-139

10. Anup Kumar and Mehmed Kantardzic: Distributed Data Mining: Framework and Implementations, IEEE Internet Computing, vol. 10, no. 4 (2006) 15-17

11. Park B. H., Kargupta H.: Distributed data mining: Algorithms, systems, and applications. The Handbook of Data Mining.Nong Ye (ed) Lawrence Erlbaum, New Jersey (2003)

12. Wallace C. S., Dowe D. L.: MML clustering of multi-state, Poisson, von Mises circular and Gaussian distributions. Statistics and Computing, 10(1) (2000) 73-83

13. Message Passing Interface Forum: MPI: A message-passing interface standard. International Journal of Supercomputer Applications, 8(3/4) (1994) 165-414

14. Quinlan, J. R.: C4.5: Programs for machine learning. San Mateo, CA: Morgan Kaufmann (1993)

15. M. Viswanathan, Y. K. Yang, T. K. Whangbo: Distributed Data Mining on Clusters with Bayesian Mixture Modeling, Lecture Notes in Computer Science, Volume 3613 (2005) $1207-1216$

16. Merz, C. and Murphy, P.: UCI repository of machine learning databases.A.B. Smith, C.D. Jones, and E.F. Roberts, "Article Title", Journal, Publisher, Location, Date (1998) 1-10 